Check for updates

Cite this: Chem. Sci., 2019, 10, 3864

๑ All publication charges for this article have been paid for by the Royal Society of Chemistry

Received 8th December 2018 Accepted 23rd February 2019

DOI: $10.1039 / \mathrm{c} 8 \mathrm{sc} 05480 \mathrm{k}$

rsc.li/chemical-science

\section{Crisscrossing coordination networks: ligand doping to control the chemomechanical properties of stimuli-responsive metallogels $\uparrow$}

\author{
Junghwan Kim and Dongwhan Lee (D) *
}

\begin{abstract}
Metallogels respond to external stimuli by changing their mechanical properties. To gain a fine control over this phase-shifting event, we have designed and introduced intentional structural mismatches into the otherwise tightly knit metal-organic networks. Built using biphenolate-derived multidirectional/ multidentate ligands, these soft materials display markedly different rheological properties depending on the level of "ligand doping", as well as the type of metal ion serving as a key structural support. A zinc metallogel optimized through this process responds to acids, both in the gas stream and liquid phase, by a rapid gel-sol transition and visually discernible colour change.
\end{abstract}

\section{Introduction}

Metal-organic networks are spontaneous assemblies of metal ions and multi-nucleating ligands to form higher-order architectures. Representative examples include metal-organic frameworks (MOFs) $)^{1-6}$ and metallogels. ${ }^{7-22}$ Here, the reversible nature of metal-ligand bonding is exploited to achieve longrange structural ordering beyond the immediate coordination sphere through self-correcting and self-reinforcing processes.

Unlike crystalline MOFs, metallogels are soft amorphous materials. Through entanglement and cross-linking of extended metal-ligand networks, however, they acquire sufficient mechanical strength to entrap and bear the weight of solvent molecules inside. While metallogels could undergo sol-gel transitions just like simple organic gelators, ${ }^{23-27}$ the presence of metal centres provides an added advantage of utilizing coordination chemistry to control the mechanical properties of the bulk material. For example, if the making and breaking of metal-ligand bonds are driven by chemical stimuli, such as ligand exchange, local chemical events can be amplified to solgel phase transitions, and readily checked by a straightforward "tube inversion test". ${ }^{8-12,14-17,23-25,27-30}$

Despite the conceptual appeal and operational simplicity, however, one critical drawback of metallogel-based chemical detection is its slow response kinetics. Specifically, the pristine gel should be sufficiently robust to withstand thermal and mechanical stress and retain its shape, but needs to

Department of Chemistry, Seoul National University, 1 Gwanak-ro, Gwanak-gu, Seoul 08826, Korea. E-mail: dongwhan@snu.ac.kr

$\dagger$ Electronic supplementary information (ESI) available: Experimental procedures and additional data $\left({ }^{1} \mathrm{H}\right.$ and ${ }^{13} \mathrm{C}$ NMR spectra, FT-IR spectra, HR-MS and elemental analysis) of the newly synthesized compounds. See DOI: $10.1039 / \mathrm{c} 8 \mathrm{sc} 05480 \mathrm{k}$ disassemble rapidly and turn into a solution upon a chemical triggering event. While proof-of-concept systems are currently available for metallogel-based chemical sensing, ${ }^{17,31-36}$ a rational

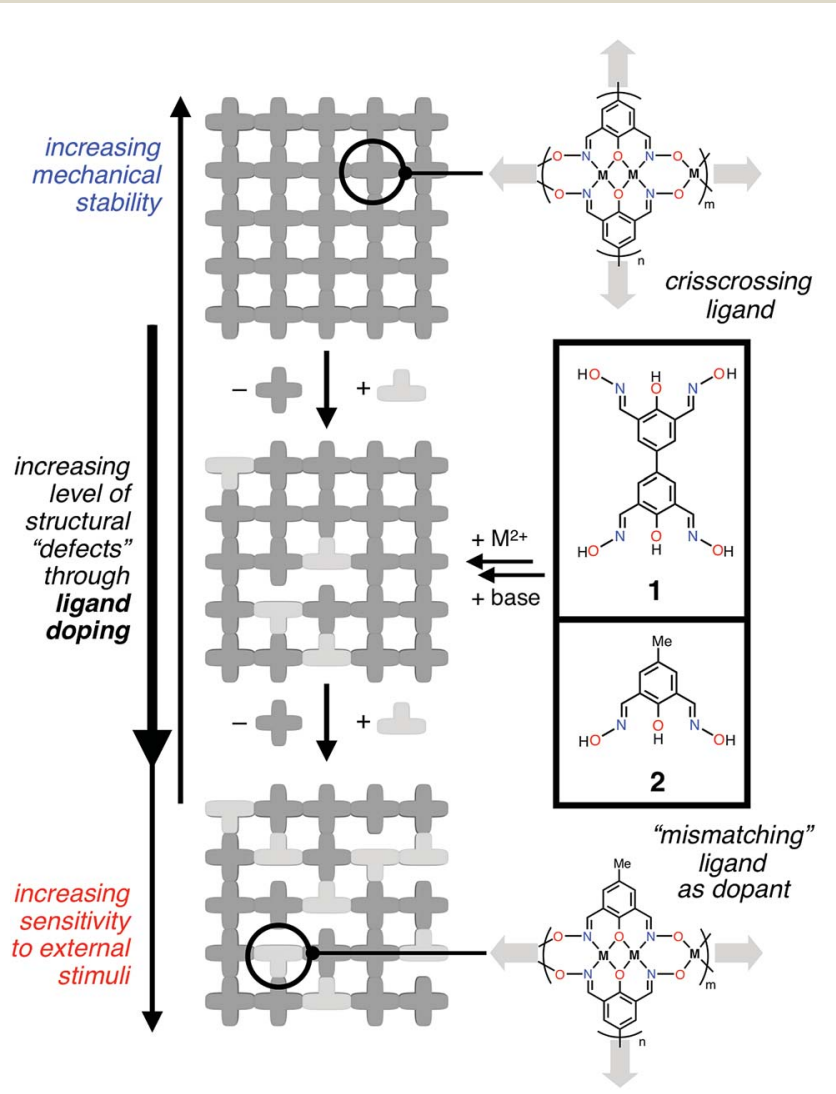

Fig. 1 Schematic representations of idealized metal-ligand networks constructed from ligand 1 and its "half-unit" derivative 2 serving as a chain terminator to create structural defects. 
design principle is needed to strike a fine balance between mechanical stability and chemical sensitivity. In this paper, we disclose our structure design and synthetic strategies to meet this important challenge (Fig. 1).

As outlined in Fig. 1, we have developed a new multinucleating chelate $\mathbf{1}$ as a fused form of the simpler half-unit ligand 2. Through binding of transition metal ions, 1 could furnish tightly knit grid-like 2D/3D networks that evolve into a gel-like structure. An intentional doping with the half-unit ligand 2, however, introduces structural "defects", which systematically decrease the viscoelasticity of the metallogel. For an optimized system, the gel-to-fluid transition is (i) induced by ligand exchange with exogenously added acid, either in solution or in the gas phase, (ii) made faster by changing either the metal centre or increasing the level of ligand doping, and (iii) monitored simultaneously by indicator molecules trapped inside the gel matrix. To the best of our knowledge, this is the first example of metallogels, in which structural doping of the ligand system controls the mechanical properties and sensory response kinetics of the bulk material. ${ }^{37-39}$ Important lessons that we have learned from this rule-finding study are discussed in the following sections.

\section{Background and design principles}

Mechanical properties of soft materials are determined by how stress dissipates through a series of chemical bonds. ${ }^{40,41}$ To finetune such properties, interconnected networks have advantages over linear structures. While a strand of polymer readily loses mechanical tension by simple bond cleavage, a strand in a network structure could still maintain the tension through cross-linking with nearby strands. According to this phantom network theory, the mechanical properties of network structures can be altered by structural defects introduced intentionally, just like doping in electronic materials. ${ }^{42-47}$ For example, functional groups of the monomers can be modified strategically to evaluate the effects of primary defects ${ }^{48-50}$ or topological defects such as loops ${ }^{48,51}$ on the elastic properties of polymer gels and elastomers.

Taking inspiration from polymer chemistry, we wished to develop metallogels having mechanical properties that are tunable by ligand design and defect doping. For this purpose, a pair of multinucleating/multidirectional ligands were needed: one participating in chain propagation, and the other functioning as a chain terminator. For this purpose, ligands $\mathbf{1}$ and $\mathbf{2}$ (Fig. 1), built upon a salicylaldoxime motif, emerged as ideal candidates.

As exemplified by the dinuclear clathrochelate complex $\mathbf{I}^{52}$ and end-capped tetranuclear complexes II and III, ${ }^{53,54}$ shown in Fig. 2, the central [N,O,N]-donor array of 2 can support phenoxide-bridged bimetallic centres. A structurally characterized trinuclear complex $\mathbf{I V}^{55}$ demonstrates that the deprotonated oximate-O atom of the simple salicylaldoxime ligand 3 (Fig. 2) could serve also as a bridge to connect two tris(chelate) mononuclear complexes by coordinating to the third metal in the middle.

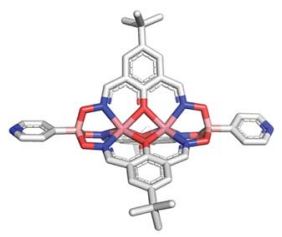

I

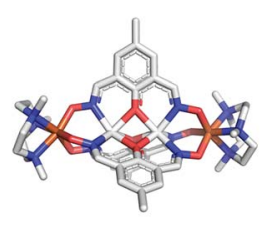

II

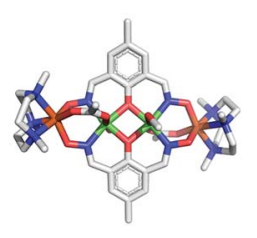

III
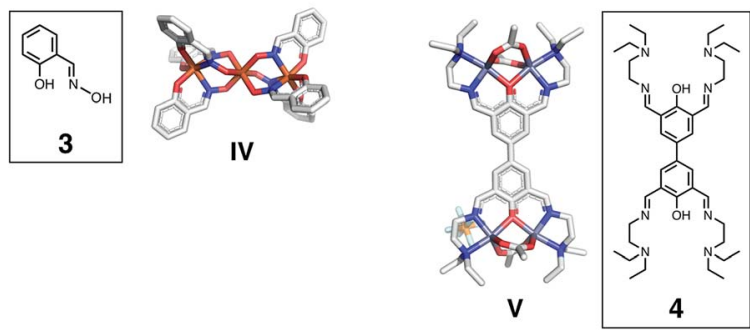

Fig. 2 Capped-stick representations of crystallographically determined polynuclear metal complexes I (CSD code: YIYQEE), ${ }^{52}$ II (CSD code: RELZAK), ${ }^{52}$ III (CSD code: ZIGQIP), ${ }^{53}$ IV (CSD code: VIFWIS), ${ }^{55}$ and $\mathrm{V}$ (CSD code: VIJQUA), ${ }^{56}$ along with the chemical structures of the ligands 3 and 4 .

To build cross-linked grids of these basic structural motifs, it is necessary to "fuse" two units of $\mathbf{2}$ into a single ligand platform 1 for bidirectional network growth through metallation (Fig. 1 and 3). A structurally related ligand $\mathbf{4}$ is known to support

(a)

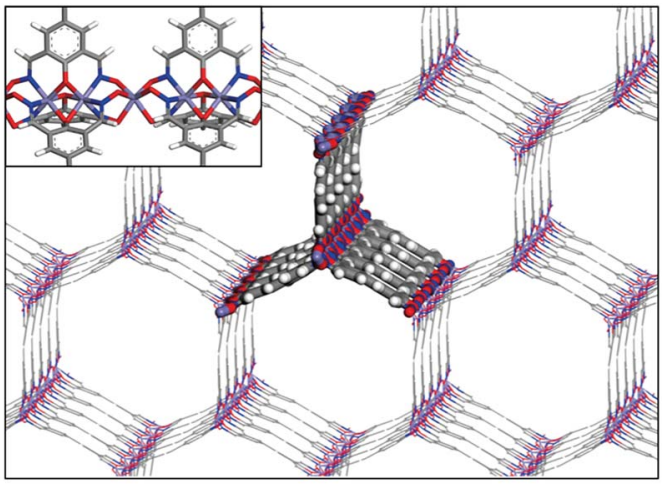

(b)

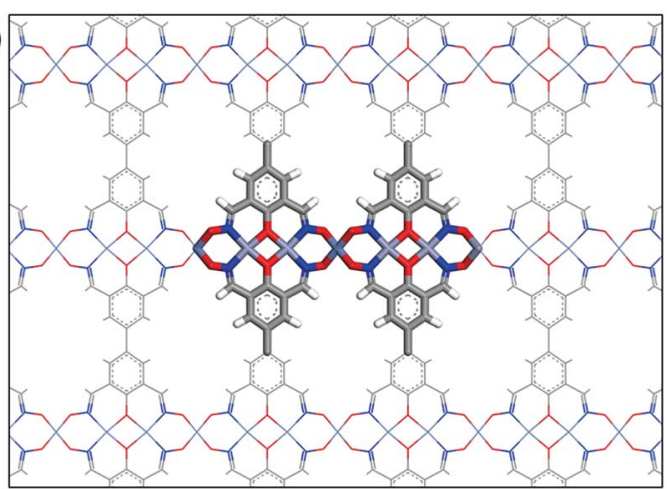

Fig. 3 Computationally generated models of the idealized metalligand network of 1 having either six-coordinate (for (a); 3D honeycomb) or four-coordinate (for (b); 2D grid) metal centres. Shown in the inset of (a) is a capped-stick model of the connected cage-like repeating unit viewed from a different angle. 
a tetranuclear complex (V, Fig. 2$),{ }^{56}$ but the chemistry of the $4,4^{\prime}$ biphenolate-derived ligand has remained surprisingly undeveloped since the initial report.

Using the structurally characterized metal complexes (Fig. 2) as starting points, we first constructed simple models of metalligand networks anticipated from 1 , either with (i) $\mathrm{M}_{2} \mathrm{~L}$ or (ii) $\mathrm{M}_{3} \mathrm{~L}$ metal-ligand stoichiometry (Fig. 3). It should be noted that these highly idealized structures could serve only as 3D skeletal models to assess the spatial arrangements and the degree of steric constraints when multiple ligand fragments are brought into close proximity by either all six-coordinate (Fig. 3a) or fourcoordinate (Fig. 3b) metal centres. In reality, the actual amorphous structure, trapped kinetically in the metallogel formation process (vide infra), would lie somewhere in between these two extremes of crystalline structural ordering. ${ }^{57,58}$ Despite such caveats, both structures modeled in Fig. 3 seemed reasonable, with no apparent interligand steric crowding or unusual coordination geometry. We thus proceeded to carry out actual synthesis to check the feasibility of the ideas proposed in Fig. 1.

\section{Results and discussion}

\section{Ligand synthesis and metallation}

The biphenol tetraoxime ligand 1 was prepared in two steps from 4,4'-biphenol (Scheme 1) via the known tetraformyl precursor $5 .{ }^{56}$ Condensation of 5 with hydroxylamine furnished 1. Using the intermediate $\mathbf{5}$, structural analogues of $\mathbf{1}$ were also prepared, which share a common biphenyl skeleton, but do not have either the terminal oxime-O donor (6) or bridging phenolate-O donor (8) groups, as shown in Scheme 1.

Metallogels of 1 were prepared in a straightforward manner by simply mixing DMF solutions of the deprotonated ligand and metal salt (Fig. 4). In a typical experiment, a $\mathrm{Zn}$ metallogel was prepared by adding a DMF solution of zinc(II) acetate into a rapidly stirred solution of $\mathbf{1}$ and $\mathrm{Et}_{3} \mathrm{~N}$. An immediate sol-to-gel transition was observed and confirmed by a simple tube inversion test (Fig. 4a). To improve the structural homogeneity, the

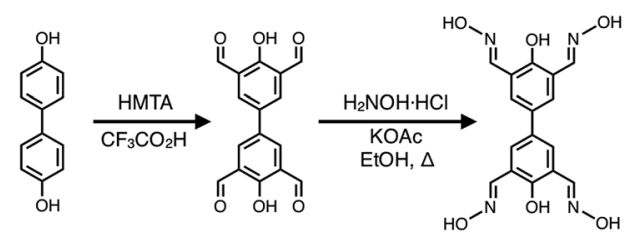

1

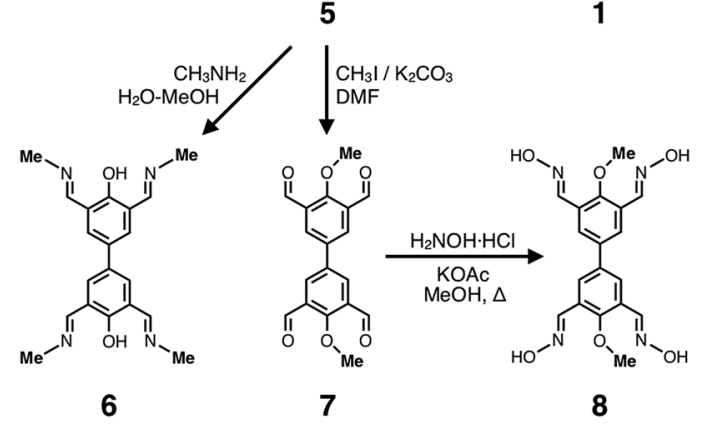

Scheme 1 Synthetic routes to ligand 1 and its derivatives.
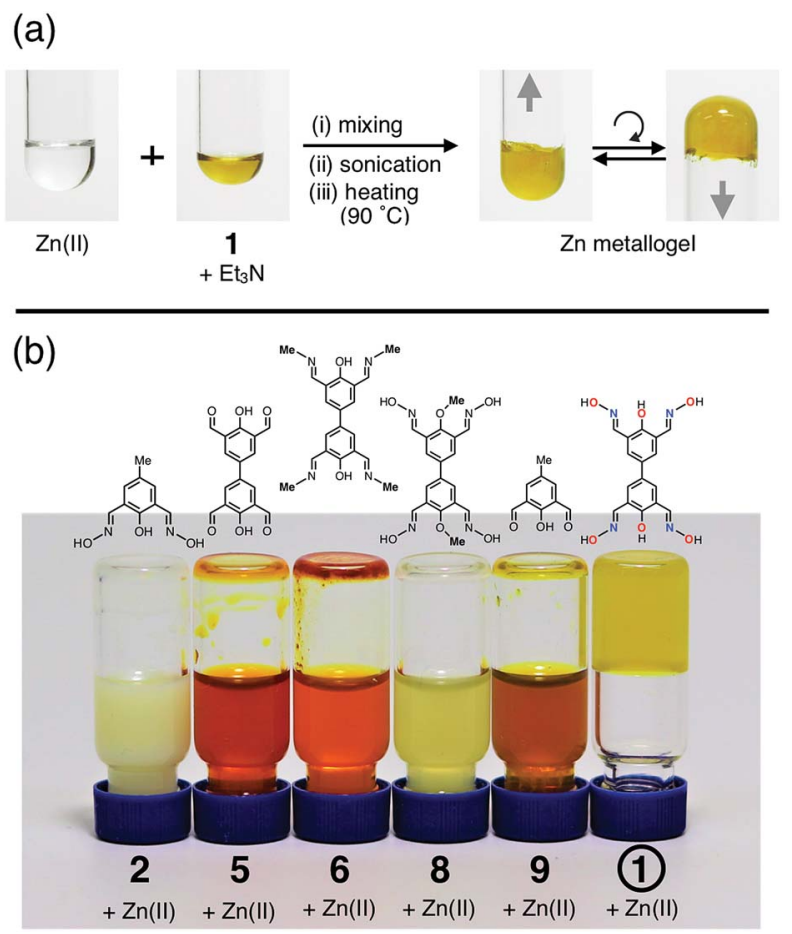

Fig. 4 (a) Formation of a zinc metallogel of 1 (4 wt\%), and (b) comparative studies with five different analogues and derivatives of 1 to identify structural parameters required for gelation.

mixture could be sonicated for $10 \mathrm{~min}$ and heated at $90{ }^{\circ} \mathrm{C}$ for several hours without structural collapse or deterioration of the gel-like properties.

Comparative studies with five structural derivatives of 1 (Fig. 4b) established that the formation of metallogels requires the presence of both (i) oximate-[N,O] and (ii) phenoxide-O donors, in conjunction with (iii) biaryl-linked extended ligand scaffolds. No gelation occurred for the truncated half-unit ligand 2, oximate-free ligands 5 and 6, phenol-protected 8, or half-unit ligand $\mathbf{9}$ without oximate, when treated with zinc(II) under identical conditions. A free-standing gel is obtained only for 1, which satisfies the structural requirements for multidirectional network growth, as modeled in Fig. 1 and 3.

\section{Morphology and spectroscopic analysis}

To investigate the morphology and internal structure of the metallogel, samples were dried with supercritical $\mathrm{CO}_{2}\left(\mathrm{SCO}_{2}\right)$ to remove unreacted starting materials and entrapped solvent molecules. Using a standard setup to dry MOF samples (Fig. S1 $\dagger$ ), the zinc metallogel (ca. $10 \mathrm{~mL}$ ) was subjected to

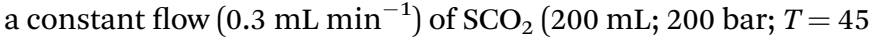
${ }^{\circ} \mathrm{C}$ ) to obtain yellow powdery aerogels (Fig. 5a).

As shown in Fig. 5b, the SEM image of the $\mathrm{Zn}$-aerogel reveals porous internal structures, in which fibrils of cross-linked metal-ligand assemblies interconnect to create mesoscopic morphologies, leaving void spaces in between. A cross-sectional TEM image (Fig. 5c) of a thinly (ca. $80 \mathrm{~nm}$ ) sliced sample, prepared by ultramicrotomy of aerogel particles, established that the porous structure spans across the bulk material, not 
(a)

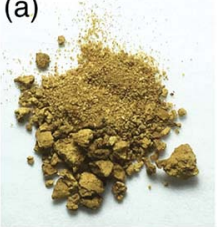

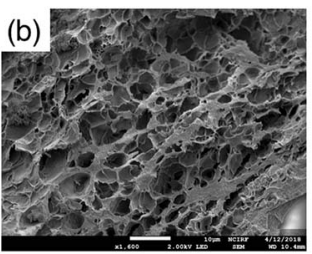

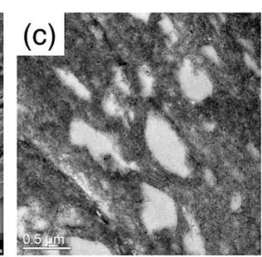

Fig. 5 (a) Photographic, (b) SEM, and (c) cross-sectional TEM images of $\mathrm{Zn}$ aerogel of 1 prepared by $\mathrm{SCO}_{2}$ drying (see text for experimental details).

being confined to the surface region. In addition, EDX analysis (Fig. S2 $\dagger$ ) confirmed an even spatial distribution of elemental C, N, O, and Zn across the entire sample. Similar observations were made for the aerogel samples of either cobalt(II) (Fig. S3 $\dagger$ ) or copper(II) (Fig. S4†) in conjunction with 1.

A strong binding of Zn(II) should be aided by deprotonation of the phenol and oxime groups of the free ligand. The IR spectrum of the free ligand 1 (Fig. S5 $\dagger$ ) has intense bands at $2700-3500 \mathrm{~cm}^{-1}$ from the $\mathrm{O}-\mathrm{H}$ stretching modes of phenol and oxime groups. In stark contrast, these features essentially disappear in the IR spectrum of the zinc aerogel (Fig. S5†), indicating that (i) the majority of the O-donor groups, either phenol or oxime, become deprotonated to bind the metal, and (ii) unreacted free ligands are not incorporated into the metallogel network and thus readily removed by $\mathrm{SCO}_{2}$ drying.

\section{Gel-to-sol phase transition responding to gas-phase chemicals}

With experimental evidence obtained for the involvement of metal-ligand bonds in the gel-like properties of the bulk material (Fig. 4, 5, and S2 $\dagger$ ), we proceeded to exploit the visually discernible sol-gel transition for gas-phase chemical detection. A logical choice of target is a volatile acid, such as $\mathrm{HCl}$, which can protonate the ligand to facilitate demetallation and provide chloride anions to assist such ligand substitution processes.

For precise measurements of the response kinetics, a controlled gas delivery system was built to ensure a constant pressure and flow rate (Fig. 6 and S6 $\dagger$ ). In each measurement, a custom-made Teflon sample holder was loaded with a fixed amount of metallogel of known wt\%, and placed inside (a)

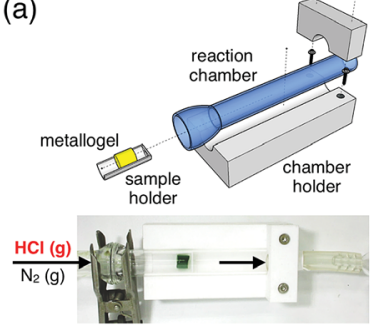

(b)

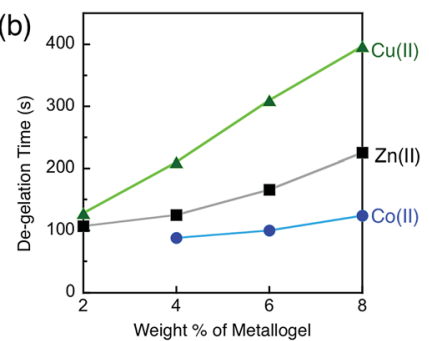

Fig. 6 (a) Schematic diagram of the controlled gas delivery system for gas-phase $\mathrm{HCl}$ detection by metallogels, and a photographic image of the actual experimental setup. (b) Plots of degelation time as a function of wt\%. Metallogels of different transition metal ions were exposed to a constant flow $\left(0.5 \mathrm{~L} \mathrm{~min}^{-1}\right)$ of $\mathrm{HCl}\left(5 \%, \mathrm{v} / \mathrm{v}\right.$, diluted in $\left.\mathrm{N}_{2}\right)$ at r.t. a transparent glass vessel. The entire unit was assembled, and connected to the gas delivery system (Fig. 6a). The reaction was initiated by delivery of gaseous $\mathrm{HCl}\left(5 \%, \mathrm{v} / \mathrm{v}\right.$, diluted in $\left.\mathrm{N}_{2}\right)$ to the reaction chamber at a constant flow rate of $0.5 \mathrm{~L} \mathrm{~min}^{-1}$; the progress of the reaction was monitored by taking digital images at fixed time intervals (Fig. S7†). A small hole was made at the bottom of the Teflon sample holder to facilitate the drainage of the solutionized sample, which marked the completion of the reaction as well.

As plotted in Fig. 6b, the gel-to-sol transition takes a longer time as the $\mathrm{wt} \%$ of the gelating material increases. This general and anticipated trend was observed consistently with the metallogels prepared with cobalt(II), copper(II), and zinc(II). At a fixed wt\%, however, the response rates are remarkably different depending on the metal of choice, despite the fact that they all share the same ligand $\mathbf{1}$. Intriguingly, the experimentally observed order of robustness, copper(II) $>$ zinc(II) $>$ cobalt(II), correlates nicely with the Irving-Williams series of the relative stabilities of first-row transition metal complexes. ${ }^{59}$

While care should be taken in interpreting the behaviour of the amorphous gel materials using simple $\mathrm{M}-\mathrm{L}$ bonding models alone, our experimental results convincingly demonstrate the practical utility of metallogels for gas-phase chemical detection, and the tunability of the response kinetics by varying the metal ion.

\section{Reversible sol-gel transition and colour switching}

If the gel-to-sol transition can be triggered by an acid (Fig. 6), it should be feasible to reverse this process by "back-titration" with a base. The majority ( $>95 \mathrm{wt} \%$ ) of the gel volume is taken up by the solvent, which could serve as a fluidic reservoir for non-escaping reporter molecules that can simultaneously track this process. In Fig. 7 is demonstrated how this dual mode of response, as a combination of phase transition and colourimetric change, is implemented with the $\mathrm{Zn}$ metallogel.

With no d-d or CT-type optical transitions to interfere with the encapsulated $\mathrm{pH}$ indicators, zinc(II) is an ideal metal of choice to build the gelating network for such purposes. An added bonus is the kinetic lability, as well as the stereochemical promiscuity of the $\mathrm{d}^{10}$ metal, which ensures rapid

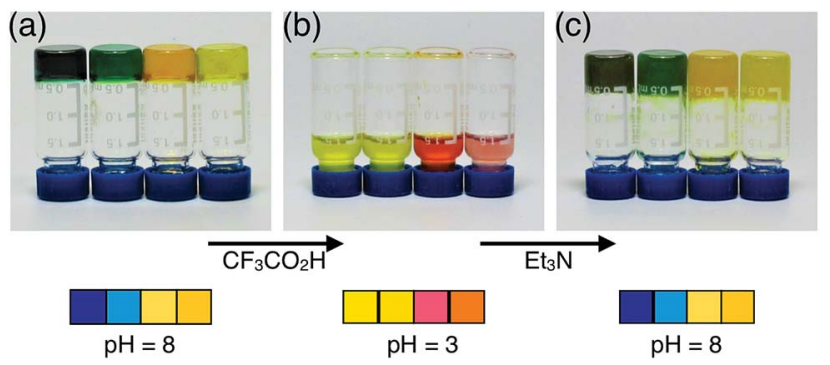

Fig. 7 Reversible sol-gel transitions of (a) the as-prepared zinc metallogels (4 wt\%) by (b) treatment with $\mathrm{CF}_{3} \mathrm{CO}_{2} \mathrm{H}$, and (c) subsequent back titration with $\mathrm{Et}_{3} \mathrm{~N}$ at r.t. Each sample was prepared using $\mathrm{pH}$ indicator molecules (bromophenol blue, bromocresol green, methyl orange, and thymol blue; from left to right) as co-gelators, the colour of which at a given $\mathrm{pH}$ (in aq solution) is provided below as a reference. 
ligand exchange that is crucial for reversible phase switching under ambient conditions. In contrast, a cobalt(II)- or copper(II)-based system shows intense transitions in the visible region, which interfere with the colourimetric sensory response.

As a proof-of-concept, $\mathrm{Zn}$ metallogels (4 wt\%) were prepared by simply adding representative $\mathrm{pH}$ indicators, such as bromophenol blue, bromocresol green, methyl orange, and thymol blue, to the reaction batch. As shown in Fig. 7a, the presence of these reporter molecules did not affect the formation of metallogels, which, upon treatment with $\mathrm{CF}_{3} \mathrm{CO}_{2} \mathrm{H}$, underwent an immediate gel-to-sol transition with a concomitant colour change (Fig. 7b). The reversible nature of this process is established by restoration of the initial gel state as well as the indicator colour, following treatment with $\mathrm{Et}_{3} \mathrm{~N}$ (Fig. 7c). By increasing the initial concentration of the sample to $12 \%$, this reversible sol-gel switching could be repeated at least six times, although its cyclability is practically limited by the dilution effect.

\section{Ligand doping to control the mechanical properties of the bulk material}

The gel-sol transition rate is a critical parameter to improve the performance of metallogels as a chemical sensor. In the metallogel, the cross-linked fibrous backbone ( $<5 \mathrm{wt} \%)$ supports the weight of the entire matrix (>95 wt\%) against gravity. By controlling the mechanical properties of the metal-ligand network, degelation kinetics could be adjusted to the desired sensitivity.

While we have demonstrated that the choice of metal ions can have a profound impact on the mechanical stability of the metallogels (Fig. 6b), the ligand is another important structural component that can be engineered to fine-tune the viscoelastic properties of the bulk material. For crystalline MOFs, one type of ligand can partially be replaced with another type of ligand. Typical strategies include solid-solution mixing or postsynthetic modification to enhance sorption, catalytic, electrical, or magnetic properties of the doped crystalline materials. ${ }^{60-68}$ In the polymer network synthesis, monomers having different numbers of functional groups can be mixed in different ratios to create defects, thereby controlling the mechanical properties of the bulk material. ${ }^{51,69-72}$ Considering the close relevance of metallogels to MOFs (in terms of structural components) and organic polymer gels (in terms of physico-mechanical properties), it is surprising that ligand doping has rarely been exploited to control the viscoelastic properties of the bulk material. ${ }^{37-39}$

Apparently, this is a challenging task. Even our own results (Fig. 4) show that a seemingly minor structural modification of the gelating motif $\mathbf{1}$, either in the ligand backbone (i.e. 2 ) or metal-binding groups (i.e. 5, 6, 8, and 9), fails to produce metallogels; the reaction either remains fluidic or simply turns into precipitates. Instead of a trial-and-error approach, we decided to adopt a systematic strategy of introducing the half-unit ligand $\mathbf{2}$ as a chain-terminating agent to suppress network growth. A total of 11 samples were thus prepared (Fig. 8), in which the ratio between the ligands 1 and 2 was varied

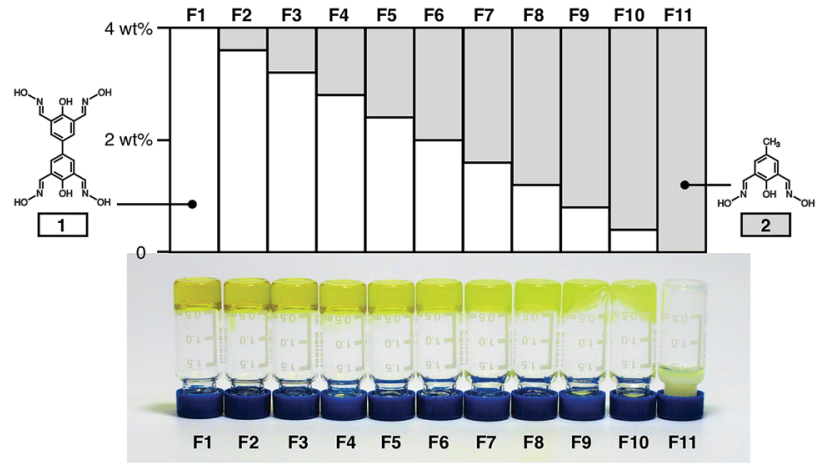

Fig. 8 Formulation of zinc metallogels F1-F11 prepared with ligands 1 and 2 in varying wt\% (top), and photographic images of the 11 samples upon a tube inversion test (bottom).

systematically while maintaining the overall "amount" of the ligand donor groups constant across the entire set.

As shown in Fig. 8, an increasing level of ligand doping of 2 (in gray bar) into $\mathbf{1}$ (in white bar) results in a gradual attenuation of gelating properties, as visually inspected by a simple inversion test. While F1-F6 maintain a gel-like behaviour, the mixture either has untrapped solvent escaping the gel matrix, or flows down the vertical wall beyond F7 (1:2=4:6, wt/wt). For a more precise, quantitative measure of the viscoelastic properties as a function of the ligand doping level, we proceeded to carry out rheological studies.

Using a standard setup, metallogel samples were placed between two plates, and their viscoelastic behaviour was measured in response to the oscillatory deformation in the range of $\omega=10^{-1}$ to $10^{2} \mathrm{rad} \mathrm{s}^{-1}$ (Fig. 9a and b). For a side-byside comparison of the formulations F1-F7, data points at $\omega$ $=10 \mathrm{rad} \mathrm{s}^{-1}$ are plotted. As shown in Fig. 9c and S8, $\dagger$ both the storage modulus and loss modulus decrease systematically with an increasing level of ligand doping. With structural defects introduced by network-terminating ligand 2, the metal-ligand network becomes less ordered, and less effective in dissipating energy under mechanical stress. This result is consistent with the qualitative trend observed in Fig. $8 .^{73}$

To confirm that doping with ligand 2 is indeed responsible for the attenuation of the mechanical properties of the metallogels (Fig. 8 and 9), we have converted samples F1-F7 to the corresponding aerogels by $\mathrm{SCO}_{2}$ drying. As shown in Fig. $\mathrm{S} 9, \dagger$ the IR spectra of powdery aerogels F1-F7 are all similar, and consistently show a lack of intense features in the 2700$3500 \mathrm{~cm}^{-1}$ energy window corresponding the phenol/oxime $\mathrm{O}-\mathrm{H}$ vibrations of the free ligands. To quantify the amount of each ligand that is actually incorporated into the metal-ligand network, each aerogel sample was subjected to acid hydrolysis for complete structural disassembly. From the mixture were isolated free ligands 1 and 2, which were analysed by ${ }^{1} \mathrm{H}$ NMR spectroscopy. As shown in Fig. 10a, the ${ }^{1} \mathrm{H}$ NMR spectrum of each sample shows well-resolved proton resonances from the imine and phenyl ring portions of the ligands $\mathbf{1}$ and 2, which aided quantification by peak integration. The batch-to-batch reproducibility of this ligand composition analysis was 

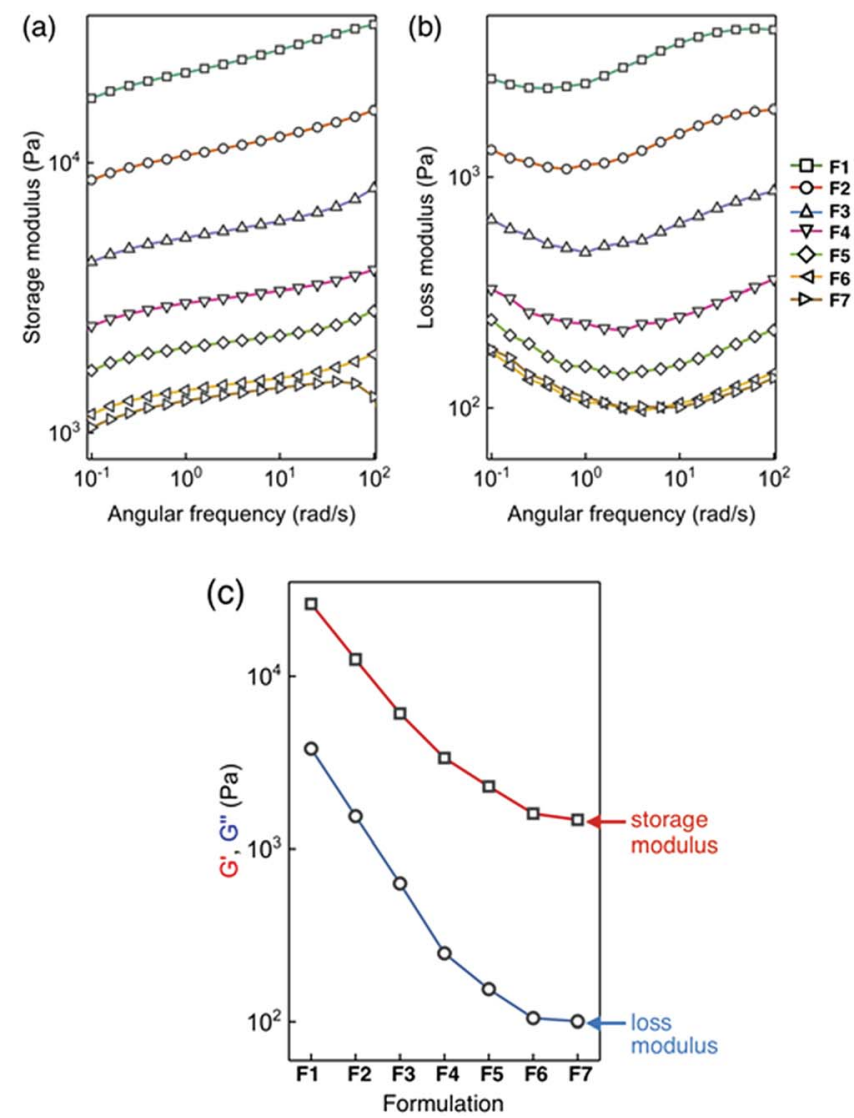

Fig. 9 (a) Storage modulus and (b) loss modulus of zinc metallogels F1-F7. In (c) are plotted storage and loss moduli determined at $\omega=$ $10 \mathrm{rad} \mathrm{s}^{-1}$

established by ${ }^{1} \mathrm{H}$ NMR (Fig. S10†) and HPLC (Fig. S11†) analysis of the representative aerogel samples, prepared with $25 \%$, $50 \%$, and $75 \%$ of the mismatching ligand 2 as the dopant. Each formulation was divided into three individual batches, which were subjected independently to acid digestion, and analysed by integration of ligand proton resonances (Fig. S10†) and HPLC peaks (Fig. S11†) to confirm a consistent ratio of $\mathbf{1}$ and $\mathbf{2}$ for the given formulations.

A plot of the mol\% of the recovered ligand $2 \mathrm{vs}$. formulations (from $\mathbf{F 1}$ to $\mathbf{F} 7$ ) shows a systematic increase and gradual saturation in the \% incorporation of the ligand 2 into the metallogel network (Fig. 10b). The formation of a gel-like structure should require a critical network density, which is largely maintained by the ligand 1. An increasing fraction of the mismatching ligand 2 would produce fragmented strands and incomplete cross-junctions that do not reach the critical network density and are thus removed in the process of $\mathrm{SCO}_{2}$ drying. As a consequence, a non-proportional relationship exists between the feed ratio of the monomers (i.e. 1 vs. 2) and their actual incorporation into the aerogel material obtained. This trend is what is observed experimentally in Fig. 10b.

Comparative powder X-ray diffraction studies on mixedligand metallogels (Fig. S12 $\dagger$ ) revealed qualitatively similar patterns in terms of the relative intensities and $2 \theta$ values. Their broad and weak diffraction features indicate the absence of (a)

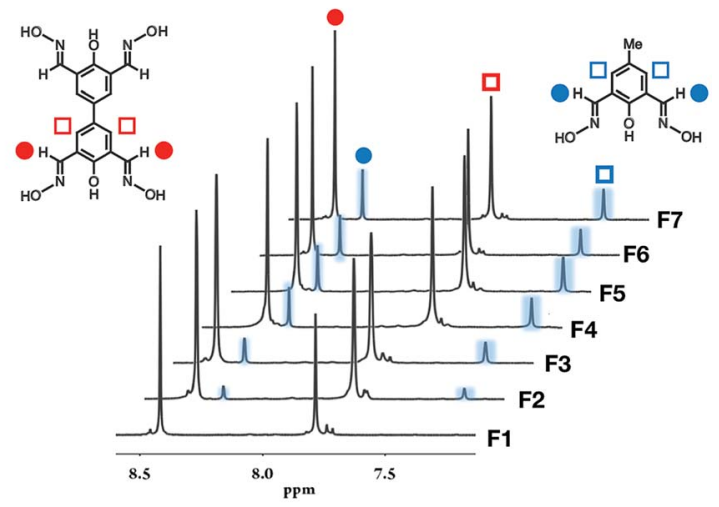

(b)

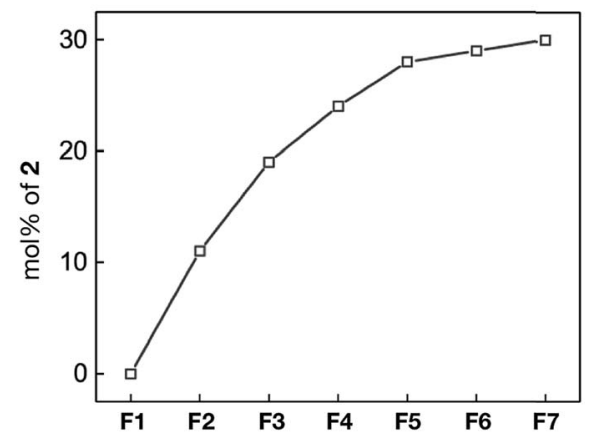

Fig. 10 (a) Partial ${ }^{1} \mathrm{H}$ NMR spectra of the acid-digestion products of the aerogel formulations F1-F7, along with peak assignments. (b) A plot of the relative composition (in mol\%) of the doping ligand 2 in F1-F7.

long-range atomic ordering and little crystallinity, as anticipated from the kinetically trapped nature of the metal-ligand assembly. More importantly, these PXRD patterns are distinctively different from the intense and well-resolved features obtained from the metal precursor $\mathrm{Zn}(\mathrm{acac})_{2} \cdot x \mathrm{H}_{2} \mathrm{O}$ or the free ligands 1 and 2 (Fig. S12†). No residual starting material, either an unreacted metal salt or a non-coordinated ligand, is thus left behind after metallogel assembly and $\mathrm{SCO}_{2}$ washing.

A uniform distribution of the metal and the ligands $\mathbf{1}$ and $\mathbf{2}$ across the bulk material was also established by EDX analysis of mixed-ligand metallogels (Fig. S13†). To ascertain batch-tobatch reproducibility, the bulk material was divided into three separate samples and analysed individually, in a manner similar to the ligand composition analyses by ${ }^{1} \mathrm{H}$ NMR spectroscopy (Fig. S10 $\dagger$ ) and HPLC (Fig. S11†). Collectively, these results support the notion that an increasing amount of network-terminating "dopant" 2 , which is randomly incorporated at the microscopic molecular level, yet evenly distributed at the macroscopic level, is indeed responsible for the decreased viscoelasticity of the bulk material (Fig. 9).

\section{Mechanical strength and degelation response rate}

With a close correlation established between the level of ligand doping and mechanical stability of the metallogels (Fig. 8 and 9), we proceeded to investigate its functional relevance. Specifically, we wished to test whether an attenuated mechanical stability, designed with intention, would indeed translate directly to an enhanced sensitivity toward chemical stimuli. 
(a)

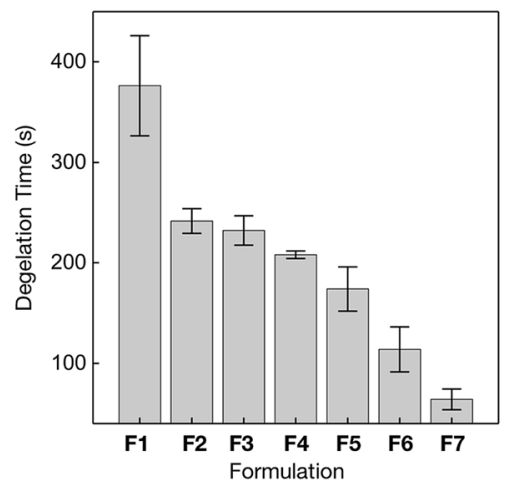

(b)

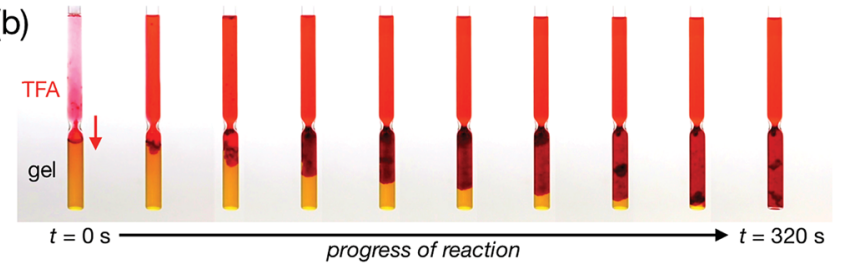

Fig. 11 (a) Plots of the average degelation time of ligand-doped metallogel samples F1-F7, and (b) time-lapse photographic images of a representative experimental setup of $\mathrm{F} 1$ taken at r.t.

To exploit the acid-triggered degelation (Fig. 6) and colourimetric monitoring with entrapped $\mathrm{pH}$ indicators (Fig. 7), a simple experimental setup was devised. As shown in Fig. 11, gravity-driven mixing and diffusion of the upper-layer acid (trifluoroacetic acid) solution into the bottom-layer metallogel was monitored by taking digital images at fixed time intervals. A marked $\mathrm{pH}$ difference between the two layers provides an excellent colour contrast to follow the progressive advance of the acid front into the basic gel matrix over time until a complete structural collapse is reached.

Metallogel samples F1-F7 were prepared as in Fig. 8. For each sample, measurements were carried out six times, and the average value and standard deviation plotted. As shown in Fig. 11a, the rate of acid-triggered degelation correlates well with structural defects; the system $\mathbf{F 7}$ with the highest level of ligand doping responds $>6$ times faster than the undoped system F1. With an optimal level of doping, F7 is barely strong enough to withstand gravity and maintains gel-like properties under ambient conditions, but collapses rapidly upon acidification. Considering that $>95 \mathrm{wt} \%$ of the gel is taken up by the solvent, the difference in the response rate is best explained by the intrinsic chemomechanical properties of the metal-ligand network itself, rather than disparate diffusion of the acid through the matrix.

For the undoped system F1, the acid-triggered degelation time remains essentially constant when the ligand-metal ratio is varied from $1: 3$ to $1: 2$, but drops significantly ( $>3$ times) when the metal ion is depleted down to a $1: 1$ ratio (Fig. S14 $\dagger$ ). This empirical observation is consistent with the structural model postulated in Fig. 3. A decrease in the amount of the metal ion below the ideal $\mathrm{M}_{2} \mathrm{~L}$ or $\mathrm{M}_{3} \mathrm{~L}$ stoichiometry would result in fragmented strands or incomplete cross-junctions compromising mechanical strength. Above the ligand-metal ratio of $1: 3$, on the other hand, the excess amount of metal ions would remain uncoordinated or exchange with ligand-bound metal centres, without significantly affecting the stability of the existing network.

\section{Conclusions}

Metallogels were constructed by using a pair of ligands that function as chain-propagating and chain-terminating groups in the network growth. These soft materials undergo chemically driven gel-sol transitions at either the liquid-gel or the air-gel interface, the response kinetics of which can be controlled by changing either the metal centre or the level of ligand doping. Collectively, our findings highlight the importance of rational ligand design and systematic compositional tuning of the metallogels to strike the right balance between physical stability and chemical sensitivity, all realised by a single system at the material level. Efforts are currently underway to generalise and refine this concept with other types of ligands, and expand the scope of signal transduction schemes to metallogel-based sensors.

\section{Conflicts of interest}

There are no conflicts to declare.

\section{Acknowledgements}

This work was supported by the Samsung Science and Technology Foundation (SRFC-MA1502-02).

\section{Notes and references}

1 S. Kaskel, The Chemistry of Metal-Organic Frameworks, 2 Volume Set: Synthesis, Characterization, and Applications, Wiley-VCH, Weinheim, 2016.

2 C. H. Hendon, A. J. Rieth, M. D. Korzyński and M. Dincă, ACS Cent. Sci., 2017, 3, 554-563.

3 H.-C. Zhou, J. R. Long and O. M. Yaghi, Chem. Rev., 2012, 112, 673-674.

4 C. K. Brozek and M. Dincă, Chem. Soc. Rev., 2014, 43, 54565467.

5 V. Guillerm, D. Kim, J. F. Eubank, R. Luebke, X. Liu, K. Adil, M. S. Lah and M. Eddaoudi, Chem. Soc. Rev., 2014, 43, 61416172.

6 H. Furukawa, K. E. Cordova, M. O'Keeffe and O. M. Yaghi, Science, 2013, 341, 1230444.

7 Y. Yu, Y. Shi and B. Zhang, Acc. Chem. Res., 2018, 51, 17111721.

8 O. Kotova, R. Daly, C. M. G. dos Santos, M. Boese, P. E. Kruger, J. J. Boland and T. Gunnlaugsson, Angew. Chem., Int. Ed., 2012, 51, 7208-7212.

9 P. Dastidar, S. Ganguly and K. Sarkar, Chem.-Asian J., 2016, 11, 2484-2498.

10 Y.-R. Liu, L. He, J. Zhang, X. Wang and C.-Y. Su, Chem. Mater., 2009, 21, 557-563.

11 A. J. McConnell, C. S. Wood, P. P. Neelakandan and J. R. Nitschke, Chem. Rev., 2015, 115, 7729-7793. 
12 M.-O. M. Piepenbrock, G. O. Lloyd, N. Clarke and J. W. Steed, Chem. Rev., 2010, 110, 1960-2004.

13 A. Mehdi, C. Reye and R. Corriu, Chem. Soc. Rev., 2011, 40, 563-574.

14 A. Y. Tam and V. W. Yam, Chem. Soc. Rev., 2013, 42, 15401567.

15 M. Martínez-Calvo, O. Kotova, M. E. Möbius, A. P. Bell, T. McCabe, J. J. Boland and T. Gunnlaugsson, J. Am. Chem. Soc., 2015, 137, 1983-1992.

16 Y. Sun, S. Li, Z. Zhou, M. L. Saha, S. Datta, M. Zhang, X. Yan, D. Tian, H. Wang, L. Wang, X. Li, M. Liu, H. Li and P. J. Stang, J. Am. Chem. Soc., 2018, 140, 3257-3263.

17 X. Yan, T. R. Cook, J. B. Pollock, P. Wei, Y. Zhang, Y. Yu, F. Huang and P. J. Stang, J. Am. Chem. Soc., 2014, 136, 4460-4463.

18 R. R. Salunkhe, Y. V. Kaneti, J. Kim, J. H. Kim and Y. Yamauchi, Acc. Chem. Res., 2016, 49, 2796-2806.

19 X. Cao, C. Tan, M. Sindoro and H. Zhang, Chem. Soc. Rev., 2017, 46, 2660-2677.

20 T. Kitao, Y. Zhang, S. Kitagawa, B. Wang and T. Uemura, Chem. Soc. Rev., 2017, 46, 3108-3133.

21 Q. Yang, Q. Xu and H.-L. Jiang, Chem. Soc. Rev., 2017, 46, 4774-4808.

22 A. Carné-Sánchez, G. A. Craig, P. Larpent, T. Hirose, M. Higuchi, S. Kitagawa, K. Matsuda, K. Urayama and S. Furukawa, Nat. Commun., 2018, 9, 2506-2513.

23 J. H. van Esch and B. L. Feringa, Angew. Chem., Int. Ed., 2000, 39, 2263-2266.

24 P. Terech and R. G. Weiss, Chem. Rev., 1997, 97, 3133-3160.

25 L. E. Buerkle and S. J. Rowan, Chem. Soc. Rev., 2012, 41, 6089-6102.

26 M. de Loos, B. L. Feringa and J. H. van Esch, Eur. J. Org. Chem., 2005, 3615-3631.

27 K. Hanabusa and M. Suzuki, Polym. J., 2014, 46, 776-782.

28 A. R. Hirst, B. Escuder, J. F. Miravet and D. K. Smith, Angew. Chem., Int. Ed., 2008, 47, 8002-8018.

29 M. Häring and D. D. Díaz, Chem. Commun., 2016, 52, 1306813081.

30 J. W. Steed, Chem. Commun., 2011, 47, 1379-1383.

31 P. Biswas, S. Ganguly and P. Dastidar, Chem.-Asian J., 2018, 13, 1941-1949.

32 W. Miao, L. Zhang, X. Wang, H. Cao, Q. Jin and M. Liu, Chem.-Eur. J., 2013, 19, 3029-3036.

33 P. Chen, Q. Li, S. Grindy and N. Holten-Andersen, J. Am. Chem. Soc., 2015, 137, 11590-11593.

34 S. Sarkar, S. Dutta, S. Chakrabarti, P. Bairi and T. Pal, ACS Appl. Mater. Interfaces, 2014, 6, 6308-6316.

35 Q. Lin, T. T. Lu, X. Zhu, B. Sun, Q. P. Yang, T. B. Wei and Y. M. Zhang, Chem. Commun., 2015, 51, 1635-1638.

36 S. Bhattacharya, S. Sengupta, S. Bala, A. Goswami, S. Ganguly and R. Mondal, Cryst. Growth Des., 2014, 14, 2366-2374.

37 Polymer-appended multidentate ligands have previously been used to study the effects of network defect sites on the diffusive motions of entrapped fluorescent labels as spectroscopic "tracers". ${ }^{\mathbf{3 8 , 3 9}}$

38 A. Habicht, S. Czarnecki, T. Rossow and S. Seiffert, J. Polym. Sci., Part B: Polym. Phys., 2017, 55, 19-29.
39 T. Rossow, A. Habicht and S. Seiffert, Macromolecules, 2014, 47, 6473-6482.

40 A. Vintiloiu and J.-C. Leroux, J. Controlled Release, 2008, 125, 179-192.

41 G. M. Kavanagh and S. B. Ross-Murphy, Prog. Polym. Sci., 1998, 23, 533-562.

42 E. R. Duering, J. Chem. Phys., 1994, 101, 8169-8192.

43 K. Oh, J. Oh, H. Choi and Y. Bae, Macromolecules, 1998, 31, 7328-7335.

44 M. Rubinstein and S. Panyukov, Macromolecules, 1997, 30, 8036-8044.

45 M. Rubinstein and S. Panyukov, Macromolecules, 2002, 35, 6670-6686.

46 P. J. Flory, Polym. J., 1985, 17, 1-12.

47 P. J. Flory, Proc. R. Soc. A, 1976, 351, 351-380.

48 H. Zhou, E.-M. Schön, M. Wang, M. J. Glassman, J. Liu, M. Zhong, D. Díaz Díaz, B. D. Olsen and J. A. Johnson, J. Am. Chem. Soc., 2014, 136, 9464-9470.

49 A. L. Andrady, M. A. Llorente, M. A. Sharaf, R. R. Rahalkar, J. E. Mark, J. L. Sullivan, C. U. Yu and J. R. Falender, J. Appl. Polym. Sci., 1981, 26, 1829-1836.

50 K. Müllen, Nat. Rev. Mater., 2016, 1, 15013-15014.

51 M. J. Zhong, R. Wang, K. Kawamoto, B. D. Olsen and J. A. Johnson, Science, 2016, 353, 1264-1268.

52 M. Pascu, M. Marmier, C. Schouwey, R. Scopelliti, J. J. Holstein, G. Bricogne and K. Severin, Chem.-Eur. J., 2014, 20, 5592-5600.

53 C. Krebs, M. Winter, T. Weyhermüller, E. Bill, K. Wieghardt and P. Chaudhuri, Chem. Commun., 1995, 1913-1915.

54 S. Khanra, T. Weyhermuller, E. Bill and P. Chaudhuri, Inorg. Chem., 2006, 45, 5911-5923.

55 M. Holynska, R. Clerac, T. Langer, R. Pottgen and S. Dehnen, Polyhedron, 2013, 52, 1425-1430.

56 E. Asato, M. Chinen, A. Yoshino, Y. Sakata and K. Sugiura, Chem. Lett., 2000, 678-679.

57 J. K. H. Hui and M. J. MacLachlan, Coord. Chem. Rev., 2010, 254, 2363-2390.

58 Q. Liu, Y. Wang, W. Li and L. Wu, Langmuir, 2007, 23, 82178223.

59 H. Irving and R. J. P. Williams, J. Chem. Soc., 1953, 31923210.

60 Z. Fang, B. Bueken, D. E. De Vos and R. A. Fischer, Angew. Chem., Int. Ed., 2015, 54, 7234-7254.

61 O. Kozachuk, I. Luz, F. X. Llabrés i Xamena, H. Noei, M. Kauer, H. B. Albada, E. D. Bloch, B. Marler, Y. Wang, M. Muhler and R. A. Fischer, Angew. Chem., Int. Ed., 2014, 53, 7058-7062.

62 R. Ameloot, M. Aubrey, B. M. Wiers, A. P. Gómora-Figueroa, S. N. Patel, N. P. Balsara and J. R. Long, Chem.-Eur. J., 2013, 19, 5533-5536.

63 P. Deria, J. E. Mondloch, O. Karagiaridi, W. Bury, J. T. Hupp and O. K. Farha, Chem. Soc. Rev., 2014, 43, 5896-5912.

64 G. Barin, V. Krungleviciute, O. Gutov, J. T. Hupp, T. Yildirim and O. K. Farha, Inorg. Chem., 2014, 53, 6914-6919.

65 Z. Fang, J. P. Dürholt, M. Kauer, W. Zhang, C. Lochenie, B. Jee, B. Albada, N. Metzler-Nolte, A. Pöppl, B. Weber, 
M. Muhler, Y. Wang, R. Schmid and R. A. Fischer, J. Am. Chem. Soc., 2014, 136, 9627-9636.

66 J. Park, Z. U. Wang, L.-B. Sun, Y.-P. Chen and H.-C. Zhou, J. Am. Chem. Soc., 2012, 134, 20110-20116.

67 S. Marx, W. Kleist and A. Baiker, J. Catal., 2011, 281, 7687.

68 M. J. Cliffe, W. Wan, X. Zou, P. A. Chater, A. K. Kleppe, M. G. Tucker, H. Wilhelm, N. P. Funnell, F.-X. Coudert and A. L. Goodwin, Nat. Commun., 2014, 5, 4176-4183.

69 D. Chan, Y. C. Ding, R. H. Dauskardt and E. A. Appel, ACS Appl. Mater. Interfaces, 2017, 9, 42217-42224.
70 K. Kawamoto, M. Zhong, R. Wang, B. D. Olsen and J. A. Johnson, Macromolecules, 2015, 48, 8980-8988.

71 K. Schwenke, M. Lang and J. U. Sommer, Macromolecules, 2011, 44, 9464-9472.

72 R. Wang, J. A. Johnson and B. D. Olsen, Macromolecules, 2017, 50, 2556-2564.

73 While Flory-Stockmayer theory is widely used to predict the gelation point of step-growth polymers, it is difficult to apply this model to our system. As shown in Fig. 4, ligand 2 forms insoluble precipitates upon metallation, which could not be characterized further to determine the "conversion of monomer groups" required for modeling. 